International Electronic Journal of Algebra

Volume 27 (2020) 206-217

DOI: $10.24330 /$ ieja.663071

\title{
ULTRA STAR OPERATIONS ON ULTRA PRODUCT OF INTEGRAL DOMAINS
}

\author{
Olivier A. Heubo-Kwegna \\ Received: 15 April 2019; Revised: 13 October 2019; Accepted: 16 October 2019 \\ Communicated by Abdullah Harmanc1
}

\begin{abstract}
We introduce the notion of ultra star operation on ultraproduct of integral domains as a map from the set of induced ideals into the set of induced ideals satisfying the traditional properties of star operations. A case of special interest is the construction of an ultra star operation on the ultraproduct of integral domains $R_{i}$ 's from some given star operations $\star_{i}$ on $R_{i}$ 's. We provide the ultra $b$-operation and the ultra $v$-operation. Given an arbitrary star operation $\star$ on the ultraproduct of some integral domains, we pose the problem of whether the restriction of $\star$ to the set of induced ideals is necessarily an ultra star operation. We show that the ultraproduct of integral domains $R_{i}$ 's is a $\star$-Prüfer domain if and only if $R_{i}$ is a $\star_{i}$-Prüfer domain for $\mathcal{U}$-many $i$.
\end{abstract}

Mathematics Subject Classification (2010): 13A15, 13A18, 16W50

Keywords: Star operation, ultraproduct of domains, Prüfer domain

\section{Introduction}

Let $R$ be an integral domain with quotient field $K$. Throughout this paper $F(R)$ denotes the set of all nonzero fractional ideals of $R$ and $f(R)$ denotes the set of all nonzero finitely generated fractional ideals of $R$.

A star operation on $R$ is a mapping $A \rightarrow A^{\star}$ of $F(R)$ into $F(R)$ such that for all $A, B \in F(R)$ and for all $a \in K \backslash\{0\}$,

(i) $(a)^{\star}=(a)$ and $(a A)^{\star}=a A^{\star}$;

(ii) $A \subseteq A^{\star}$ and $A \subseteq B \Rightarrow A^{\star} \subseteq B^{\star}$, and

(iii) $A^{\star \star}:=\left(A^{\star}\right)^{\star}=A^{\star}$.

For an overview of star operations, the reader may refer to [5, Sections 32 and 34]. An $I \in F(R)$ is a $\star$-ideal if $I^{\star}=I$. An obvious example of a star operation is the identity map traditionally denoted by $d$ and defined as $A^{d}=A$ for all $A \in$ $F(R)$. Another well known star operation we intensively use in this paper is the $v$-operation. For $A, B \in F(R),(B: A)=\{x \in K: x A \subseteq B\}$ and $A^{-1}=(R: A)$. The $v$-operation is defined by $A_{v}=A^{-1-1}$ for all $A \in F(R)$. The ideal $A_{v}$ is also called the $v$-closure of $A$. If $A_{v}=A$, then $A$ is called a divisorial ideal of $R$. Another 
key example of star operation to be used in the paper is the so called $b$-operation defined on an integrally closed domain $R$ by $A^{b}=\bigcap_{\alpha} A V_{\alpha}$ for all $A \in F(R)$, where $\left\{V_{\alpha}\right\}$ is the family of all valuation overrings of $R$.

The notion of star operation in commutative rings is often used to not only provide generalizations of classical domains, but to also produce a common treatment and deeper understanding of those domains. One of these instances is the notion of Prüfer $\star$-multiplication domain which generalizes the notion of Prüfer domains [3,6]; a notion that will be used in this paper. Under the same spirit, in this paper, we introduce the notion of ultra star operation on an ultraproduct of integral domains and provide some examples. We show that a "true" star operation on a domain does not always restrict to an ultra star operation. For easy reference, in Section 2, we review some definitions and results of ultraproducts of commutative rings that can be found in $[10,11]$ and provide some results to be used in later sections.

In Section 3, we define an ultra star operation on the ultraproduct $\mathfrak{R}$ of integral domains $R_{i}$ 's and show how to build a "star operation", called ultra star operation, from star operations on $R_{i}$ 's. We show that this construction yields the ultra $v$ operation and the ultra $b$-operation when we start with the traditional $v$-operations on the $R_{i}$ 's and the traditional $b$-operations on the $R_{i}$ 's respectively.

In Section 4 , we show as an application of the introduction of the notion of ultra star operation that if an ultra star operation $\star$ on $\mathfrak{R}$ is built from star operations

$\star_{i}$ 's on the components $R_{i}$ 's as in Proposition 3.1, then $\Re$ is a $\star$-Prüfer domain if and only if $R_{i}$ is a $\star_{i}$-Prüfer domain for $\mathcal{U}$-many $i$. We recall here that if $\mathcal{U}$ is an ultrafilter on a nonempty set $X$, a property $\mathcal{P}$ holds for $\mathcal{U}$-many $i$ if the set of all $i \in X$ such that $R_{i}$ satisfy $\mathcal{P}$ is an element of $\mathcal{U}$ (see Section 2 below).

\section{Notations and preliminaries}

Let $X$ be a set. Let $\mathcal{P}(X)$ be the collection of all subsets of $X$. A nonempty set $\mathcal{F} \subseteq \mathcal{P}(X)$ is a filter on $\mathcal{P}(X)$ if for all $A, B \in \mathcal{P}(X)$ we have:

(1) if $A, B \in \mathcal{F}$, then $A \cap B \in \mathcal{F}$;

(2) if $A \in \mathcal{F}$ and $A \subseteq B$, then $B \in \mathcal{F}$.

A filter $\mathcal{F}$ on $\mathcal{P}(X)$ is proper if it is properly contained in $\mathcal{P}(X)$. A proper filter $\mathcal{F}$ is a maximal filter if the only filter that contains it is $\mathcal{P}(X)$. By Zorn's Lemma, every proper filter of $\mathcal{P}(X)$ is contained in a maximal filter of $\mathcal{P}(X)$.

An ultrafilter $\mathcal{U}$ on $X$ is a maximal filter on $\mathcal{P}(X)$. Equivalently, a filter $\mathcal{U}$ on $X$ is an ultrafilter if and only if for all $A \subseteq X$, either $A \in \mathcal{U}$ or $X \backslash A \in \mathcal{U}$. If an ultrafilter $\mathcal{U}$ contains a finite subset of $X$, then it contains a singleton set, 
say $\{x\}$, and $x$ is an element of every element of $\mathcal{U}$. In this case $\mathcal{U}$ is a principal ultrafilter. An ultrafilter that is not principal is called a free ultrafilter. Let $\left\{R_{i}\right\}_{i \in I}$ be the collection of commutative rings indexed by a set $I$. The direct-product $R$ of the commutative rings $\left\{R_{i}\right\}_{i \in I}$ is a commutative ring consisting of elements $\left(a_{i}\right)_{i \in I}=\left(a_{1}, a_{2}, \ldots, a_{i}, \ldots\right)$, with $a_{i} \in R_{i}$.

If $\mathcal{U}$ is an ultrafilter on $I$, then we define on $R$ a relation $\sim$ by

$$
\left(a_{i}\right)_{i \in I} \sim\left(b_{i}\right)_{i \in I} \Longleftrightarrow\left\{i \in I: a_{i}=b_{i}\right\} \in \mathcal{U}
$$

The quotient set $R / \sim$ is denoted $\prod_{\mathcal{U}} R_{i}=: \mathfrak{R}$ is called the ultraproduct of the $R_{i}$ 's with respect to the ultrafilter $\mathcal{U}$. We denote simply by $\left(a_{i}\right)$ the element of $\mathfrak{R}$ determined by the equivalence class of $\left(a_{i}\right)$. In the case that for all $i \in I, R_{i}=R$, then $\mathfrak{R}$ is called the ultrapower of $R$ and is denoted $\mathfrak{R}^{\mathcal{U}}$.

2.1. Los' Theorem. A property $\mathcal{P}$ holds for $\mathcal{U}$-many $i$ if the set of all $i$ such that $R_{i}$ satisfy $\mathcal{P}$ is an element of $\mathcal{U}$. Any first order formula over the language of commutative rings utilizes the symbols $+, .,=, 0,1$. One of the most fundamental properties of ultraproducts due to Los is that they preserve first order properties.

Theorem 2.1. (Los' Theorem) Let $\phi$ be a formula in the first order language of rings. Then $R$ satisfies $\phi$ if and only if $R_{i}$ satisfies $\phi$ for $\mathcal{U}$-many $i$.

It follows from Los' Theorem that $\mathfrak{R}$ is an integral domain (a field resp.) if and only if $R_{i}$ is an integral domain (a field resp.) for $\mathcal{U}$-many $i$. This is because the theory of integral domains and fields consists of finitely many axioms, all of which can be easily be expressed in the first order language of integral domains and fields.

If $\mathcal{U}$ is a principal ultrafilter, say $\{i\} \in \mathcal{U}$, then it is not hard to see that $\mathfrak{R} \cong R_{i}$. Thus, throughout this paper, we are interested in a collection of integral domains $\left\{R_{i}\right\}_{i \in I}$ indexed by an infinite set $I$, and $\mathcal{U}$ is a non principal ultrafilter. The quotient field of each $R_{i}$ is denoted $F_{i}$. The quotient field of $\mathfrak{R}$ is the ultraproduct $\prod_{\mathcal{U}} F_{i}=: \mathfrak{F}$.

An ideal $A$ of a commutative ring $R$ is definable in $R$ if there exists a first order formula $\psi\left(x, y_{1}, \ldots, y_{n}\right)$ in the language of commutative rings and $r_{1}, \ldots, r_{n} \in R$ such that

$$
r \in A \Leftrightarrow \psi\left(r, r_{1}, \ldots, r_{n}\right) \text { is true in } R .
$$

A finitely generated ideal $A$ of a domain $R$ with quotient field $K$ and its dual $A^{-1}$ are definable, as well as the maximal ideal of any quasilocal ring (see [10]). For example, if $A:=\left(r_{1}, r_{2}, \ldots, r_{n}\right)$ is a finitely generated ideal of $R$, then $x \in K$ is in 
$A^{-1}$ if there exist some $r, \alpha_{1}, \alpha_{2}, \ldots, \alpha_{n} \in R$ such that $r=x \alpha_{1} r_{1}+x \alpha_{2} r_{2}+\ldots+$ $\left.x \alpha_{n} r_{n}\right)$. Thus $A^{-1}$ is defined in $K$ by the formula

$$
\phi\left(s, t_{1}, t_{2}, \ldots, t_{n}\right): \exists r, \alpha_{1}, \alpha_{2}, \ldots, \alpha_{n}\left(r=s \alpha_{1} t_{1}+s \alpha_{2} t_{2}+\ldots+s \alpha_{n} t_{n}\right),
$$

since $x \in A^{-1}$ if and only if $\phi\left(x, r_{1}, r_{2}, \ldots, r_{n}\right)$ is true in $K$.

Recall that an ideal $A$ of $\mathfrak{R}$ is induced if $A=\left(A_{i}\right)_{\mathcal{U}}$ for some subsets $A_{i}$ of $R_{i}$, $i \in I$. Observe that $A=\left(A_{i}\right)$ is an induced ideal of $\mathfrak{R}$ if and only if $A_{i}$ is an ideal of $R_{i}$ for $\mathcal{U}$-many $i$. Some facts about the algebra of induced ideals are provided in Proposition 2.2. Recall also that an ideal $A$ of a commutative ring is $n$-generated if $A$ can be generated by $n$ elements. Note that an ideal $A$ is $n$-generated if and only if $A=\left(E_{i}\right)_{\mathcal{U}}$ for some $n$-generated ideals $E_{i}$ of $R_{i}$.

Proposition 2.2. (See also [10, Lemma 2.1]) Suppose that $A=\left(A_{i}\right)_{\mathcal{U}}$ and $B=$ $\left(B_{i}\right)_{\mathcal{U}}$ are induced ideals of $\mathfrak{R}$. Then

(i) $A \subseteq B$ if and only if $A_{i} \subseteq B_{i}$ for $\mathcal{U}$-many $i$;

(ii) $\mathfrak{R} / A \cong \prod_{\mathcal{U}} R_{i} / A_{i}$;

(iii) $A$ is a prime (maximal) ideal if and only if $A_{i}$ is a prime (maximal) ideal of $R_{i}$ for $\mathcal{U}$-many $i$;

(iv) $A$ is an n-generated ideal if and only if $A_{i}$ is an n-generated ideal for $\mathcal{U}$ many $i$;

(v) $A \cap B=\left(A_{i} \cap B_{i}\right)_{\mathcal{U}}$;

(vi) $A^{-1}=\left(A_{i}^{-1}\right) \mathcal{U}$;

(vii) $A$ is an invertible ideal of $\Re$ if and only if there exists $n>0$ such that $A_{i}$ is an $n$-generated invertible ideal of $R_{i}$ for $\mathcal{U}$-many $i$;

(viii) $A$ is a divisorial ideal of $\mathfrak{R}$ if and only if $A_{i}$ is a divisorial ideal of $R_{i}$ for $\mathcal{U}$-many $i$.

Proof. All the statements are shown in [10, Lemma 2.1] except (vi). To see (vi), if $A=\left(A_{i}\right)_{\mathcal{U}}$, let $x=\left(x_{i}\right) \in A^{-1}$. Then $x\left(A_{i}\right)_{\mathcal{U}} \subseteq \mathfrak{R}$, that is, by (i), $x_{i} A_{i} \subseteq R_{i}$ for $\mathcal{U}$-many $i$. Thus $x_{i} \in A_{i}^{-1}$ for $\mathcal{U}$-many $i$ and so $x \in\left(A_{i}^{-1}\right) \mathcal{U}$ by Los' Theorem. Hence $A^{-1}=\left(A_{i}^{-1}\right) \mathcal{U}$.

In general, except in some very special cases, an ultraproduct of Noetherian rings is non-Noetherian (see for example [13, Page 12, 1.4.13]) with a very complicated prime spectrum. However, it is of interest in applications to understand ultraproduct of Noetherian rings. For example H. Shoutens studies in [13, Chapter 8] tight closures in positive characteristic by relying on ultraproduct of Noetherian rings.

We next assume that the $R_{i}$ 's are Noetherian and are chosen such that the ultraproduct $\mathfrak{R}$ is Noetherian. Then we aim to prove that an induced ideal $J$ of $\mathfrak{R}$ 
is integrally closed if and only if each component $J_{i}$ of $J$ is an integrally closed ideal of $R_{i}$ for $\mathcal{U}$-many $i$. Recall that for an ideal $A$ of an integral domain $R$, the integral closure $A^{b}$ of $A$ consists of $r \in R$ satisfying the integral dependence equation of $r$ over $A: r^{s}+a_{1} r^{s-1}+a_{2} r^{s-2}+\ldots+r a_{s-1}+a_{s}=0$ for some positive integer $s$ and some $a_{i} \in A^{i}$ [7, Definition 1.1.1]. Equivalently $A^{b}=\bigcap_{\alpha} A V_{\alpha}$, where $\left\{V_{\alpha}\right\}$ is the family of all valuation overrings of $R$ [5, page 398]. An ideal $A$ of $R$ is said to be integrally closed if $A^{b}=A$.

Proposition 2.3. Let us assume that the $R_{i}$ 's are Noetherian domains such that $\mathfrak{R}$ is Noetherian. Then an induced ideal $A=\left(A_{i}\right)_{\mathcal{U}}$ of $\mathfrak{R}$ is integrally closed if and only if $A_{i}$ is an integrally closed ideal of $R_{i}$ for $\mathcal{U}$-many $i$.

Proof. $(\Leftarrow)$ Suppose in one direction that the $A_{i}$ 's are integrally closed for $\mathcal{U}$ many $i$ and let us show that $A=\left(A_{i}\right)_{\mathcal{U}}$ is integrally closed. Let $f \in A^{b}$ satisfying an integral dependence equation witnessing this. Write $f$ as an ultraproduct of elements $f_{i} \in R_{i}$, then Los' Theorem gives for $\mathcal{U}$-many $i$ an integral dependence equation witnessing that $f_{i}$ lies in the integral closure of $A_{i}$, whence in $A_{i}$, and hence lies in $A=\left(A_{i}\right)_{\mathcal{U}}$.

$(\Rightarrow)$ Conversely, suppose $A:=\left(A_{i}\right)_{\mathcal{U}}$ is integrally closed but not the $A_{i}$ 's for $\mathcal{U}$-many $i$. Let's choose some $f_{i} \in R_{i}$ in the integral closure of $A_{i}$ but not in $A_{i}$ itself. Let $f_{\mathcal{U}}$ be the ultraproduct of those $f_{i}$ 's. By Los' Theorem, $f_{\mathcal{U}}$ does not lie in $A$ whence not in its integral closure as $A^{b}=A$, since $\mathfrak{R}$ is Noetherian, so that by a well-known integrality criterion (see [13, Theorem 8.4.1]), there exists a Discrete Valuation Ring (for short DVR) $V$ containing $A$ such that $f_{\mathcal{U}} \notin A V$. We may assume $V$ to be complete and with algebraic closed residue field, and then by some arguments on cataproducts (see [13, Proof of Theorem 11.1.4]), it follows that there exists for each $i$ a DVR $V_{i}$ containing $R_{i}$ such that their cataproduct $V_{\sharp}$ is equal to $V$ (recall that the cataproduct $V_{\sharp}$ is the quotient of the ultraproduct $V_{\mathcal{U}}$ modulo the intersection of the powers of the maximal ideal [13, page 172]). Hence $\mathfrak{R}$ is contained in $V_{\mathcal{U}}$ and we must have $f_{\mathcal{U}} \notin A V_{\mathcal{U}}$ (lest this membership would then also be true in the quotient $\left.V_{\sharp}=V\right)$. By Los' Theorem, $f_{i} \notin A_{i} V_{i}$ for $\mathcal{U}$-many $i$, which in turn means by the integrality criterion alluded to above that $f_{i}$ does not belong to the integral closure of $A_{i}$ (since the $R_{i}$ 's are Noetherian), a contradiction.

Remark 2.4. 1. Note that even though we assume the $R_{i}$ 's to be Noetherian, we do not need it to show the direction $(\Leftarrow)$.

2. Observe that an induced ideal $A=\left(A_{i}\right)_{\mathcal{U}}$ is integrally closed if and only if $A$ satisfies the sentence $\phi_{n}$ that asserts that if $a, b \in A$ and $f(a, b)=0$ for 
some degree $n$ and for some $f(x, y):=x^{n}+r_{n-1} x^{n-1} y+\ldots+r_{0} y^{n} \in A[x, y]$. Note that Schmidt-van den Dries theorem [13, Section 7.2] proves that the necessary bounds exist so that the integral closure of ideals would be a definable condition. And hence Los' Theorem will only prove Proposition 2.3 for ultraproduct of ideals generated by polynomials of degree $d$ (these form a strict subclass of all induced ideals). Hence definability is not always the road to go, especially that the two directions of Proposition 2.3 might need different proofs (notice that the direction $(\Leftarrow)$ of the proof cannot be reversed since we might get integral equations of increasing degree, which become meaningless in the ultraproduct).

\section{Ultra star operations on ultra product of domains}

In the star operation context, it is customary to replace the $v$-operation by an arbitrary star operation in results such as Proposition 2.2 (viii) in order to generalize concepts. Proposition 2.2 (viii) is therefore our motivation to introduce the notion of induced star operation on an ultraproduct of integral domains.

From now on assume that $\star_{i}$ is a star operation on $R_{i}$ for each $i \in I$ and denote $\operatorname{Ind}(\mathfrak{R})$ the collection of induced ideals of $\mathfrak{R}$. Defined the map:

$$
\star: \operatorname{Ind}(\Re) \rightarrow \operatorname{Ind}(\Re), A=\left(A_{i}\right)_{\mathcal{U}} \mapsto A^{\star}=\left(A_{i}^{\star i}\right) \mathcal{U} .
$$

Proposition 3.1. The map $\star$ as defined above does satisfy the following properties:

$\left(\operatorname{Ind}_{1}\right) \mathfrak{R}^{\star}=\mathfrak{R}$ and $\left(\left(a_{i}\right) A\right)^{\star}=\left(a_{i}\right) A^{\star}$;

$\left(\operatorname{Ind}_{2}\right) A \subseteq A^{\star}$ and $A \subseteq B \Rightarrow A^{\star} \subseteq B^{\star}$, and

$\left(\operatorname{Ind}_{3}\right) A^{\star \star}:=\left(A^{\star}\right)^{\star}=A^{\star}$;

for all $A, B \in \operatorname{Ind}(\mathfrak{R})$ and for all $\left(a_{i}\right) \in \mathfrak{F} \backslash\{0\}$.

Proof. Statements $\left(\operatorname{Ind}_{2}\right)$ and $\left(\operatorname{Ind}_{3}\right)$ are clear by combining axioms (ii) and (iii) of each $\star_{i}$ described in the introduction and Proposition $2.2(i)$.

For $\left(\operatorname{Ind}_{1}\right)$, note that $\mathfrak{R}=\left(R_{i}\right)_{\mathcal{U}}$ and hence $\mathfrak{R}^{\star}=\mathfrak{R}$, since $R_{i}^{\star_{i}}=R_{i}$. Note also that $\left(\left(a_{i}\right) A\right)_{\mathcal{U}}=\left(a_{i} A_{i}\right)_{\mathcal{U}}$ and it will then follow that $\left(\left(a_{i}\right) A\right)^{\star}=\left(a_{i}\right) A^{\star}$, since $\left(a_{i} A_{i}\right)^{\star_{i}}=a_{i} A_{i}^{\star_{i}}$.

We have then motivated the following definition:

Definition 3.2. $\quad$ (i) An ultra star operation $\star$ on $\mathfrak{R}$ is a mapping from $\operatorname{Ind}(\mathfrak{R})$ into $\operatorname{Ind}(\Re)$ satisfying the conditions $\operatorname{Ind}_{1}, \operatorname{Ind}_{2}$ and $\operatorname{Ind}_{3}$ in Proposition 3.1 . 
(ii) A strong ultra star operation $\star$ on $\mathfrak{R}$ is a star operation $\star$ on $\mathfrak{R}$ in the classical sense preserving induced ideals, i.e., for each induced ideal $A$ of $\mathfrak{R}$ $A^{\star}$ is an induced ideal of $\mathfrak{R}$.

Remark 3.3. It is clear that any strong ultra star operation on $\mathfrak{R}$ is an ultra star operation, but the converse is not necessarily true (see Question.)

If we are given some star operations $\star_{i}$ on $R_{i}$ for each $i$, then $\star$ constructed in Proposition 3.1 is an ultra star operation on $\mathfrak{R}$.

Example 3.4. $\quad$ 1. The identity $d$ is clearly an ultra star operation on $\mathfrak{R}$.

2. The $v$-operation on $\mathfrak{R}$ preserves the induced ideals. In fact, by Proposition $2.2(v i)$, we have $A_{v}=A^{-1-1}=\left(A_{i}^{-1-1}\right)_{\mathcal{U}}=\left(A_{i}^{v_{i}}\right) \mathcal{U}$. So the $v$-operation is a strong ultra star operation on $\mathfrak{R}$.

3. In Proposition 3.1, if each $\star_{i}=v_{i}$ for each $i$, then the mapping $A=\left(A_{i}\right)_{\mathcal{U}} \mapsto$ $A^{\star}=\left(A_{i}^{v_{i}}\right) \mathcal{U}$ is an ultra star operation that coincides with the restriction of the $v$-operation of $\mathfrak{R}$ on the set of induced ideals in view of Proposition $2.2(v i)$ and (viii). So the mapping $A=\left(A_{i}\right) \mathcal{U} \mapsto A^{\star}=\left(A_{i}^{v_{i}}\right) \mathcal{U}$ may be legitimately called the ultra $v$-operation on $\mathfrak{R}$.

4. If each $\star_{i}=b_{i}$ for each $i$, where $b_{i}$ is the integral closure of ideals of $R_{i}$, where each $R_{i}$ is assumed to be integrally closed so that each $b_{i}$ is a star operation on $R_{i}$, then the mapping $A=\left(A_{i}\right)_{\mathcal{U}} \mapsto A^{\star}=\left(A_{i}^{b_{i}}\right)_{\mathcal{U}}$ is an ultra star operation. Note that by Proposition $2.3, A^{\star}$ is an integrally closed ideal of $\mathfrak{R}$ (we do not need the assumption of the $R_{i}$ 's being Noetherian for this) and therefore can be legitimately labeled as the ultra b-operation. Also note that $\mathfrak{R}$ is integrally closed as all the $R_{i}$ 's are integrally closed. In fact, recall that a domain $R$ is integrally closed if and only if for all $n>0$, $R$ satisfies the sentence $\phi_{n}$ that asserts that if $a, b \in R$ and $f(a, b)=0$ for some degree $n$ form $f(x, y):=x^{n}+r_{n-1} x^{n-1} y+\ldots+r_{0} y^{n} \in R[x, y]$, then $b$ divides $a \in R$. Thus the statement holds by Los' Theorem.

Note however that we do not know whether the ultra $b$-operation coincide with the restriction of the $b$-operation of $\mathfrak{R}$. More generally, we do not expected all star operations on $\mathfrak{R}$ to preserve induced ideals and therefore we may not obtain an ultra star operation just by restricting any star operation of $\mathfrak{R}$ on the set of induced ideals. We can make the following observation for the $b$-operation in the case we assume that the $R_{i}$ 's are Noetherian domains such that $\mathfrak{R}$ is Noetherian: if $A=\left(A_{i}\right)_{\mathcal{U}}$ is an induced ideal of $\mathfrak{R}$, then $A^{b}=\left(A_{i}\right)_{\mathcal{U}}^{b} \subseteq\left(A_{i}^{b_{i}}\right)_{\mathcal{U}}^{b}=\left(A_{i}^{b_{i}}\right) \mathcal{U}$, the later equality holds by combining Proposition $2.2(i)$ and Proposition 2.3. If $A^{b}$ is 
induced, then $A^{b}=\left(B_{i}\right)_{\mathcal{U}}$. So $\left(A_{i}\right)_{\mathcal{U}} \subseteq\left(B_{i}\right)_{\mathcal{U}} \subseteq\left(A_{i}^{b_{i}}\right)_{\mathcal{U}}$, that is $A_{i} \subseteq B_{i} \subseteq A_{i}^{b_{i}}$ for $\mathcal{U}$-many $i$.

Question: If $A$ is an induced ideal of $\mathfrak{R}$, must $A^{b}$ be an induce ideal?

Remark 3.5. 1. The $\star_{i}$ 's in the construction of $\star$ in Proposition 3.1 are arbitrary star operations on $R_{i}$ 's. If we pick the star operation to be of the same kind (say for example, the $\star_{i}$ is the identity for all $i$ ), then the ultra star operation $\star$ obtained should be denoted $\star \mathcal{U}$. For instance $d_{\mathcal{U}}$ means the ultra star operation obtained by considering the identity on each $R_{i}$ in Proposition 3.1, $v_{\mathcal{U}}$ is the ultra $v$-operation on $\mathfrak{R}$ when the $v$-operation is considered on each $R_{i}$, and $b_{\mathcal{U}}$ is the ultra $b$-operation on $\mathfrak{R}$ when the $b$-operation is considered on each $R_{i}$.

2. Though we could not decide, even in the special case where the $R_{i}$ 's are chosen Noetherian such that $\mathfrak{R}$ is also Noetherian, if the $b$-operation is a strong ultra star operation, note that we can at least construct an ultra star operation as in Proposition 3.1 from the $b_{i}$-operations on $R_{i}$ 's without the assumption on the $R_{i}$ 's being Noetherian. So the ultra star operation $b_{\mathcal{U}}$ may not coincide with the $b$-operation on $\mathfrak{R}$.

3. From Proposition 2.3, we can choose different kinds of star operations on $R_{i}$ 's (for instance, one could randomly pick the identity, the $v$-operations, the $b$-operations at different components of the ultraproduct $\mathfrak{R})$ to built a complex ultra star operation on $\mathfrak{R}$.

\section{Ultra star operations and Prüfer $\star$-multiplication domains}

In this section we aim to establish that if an ultra star operation $\star$ on $\mathfrak{R}$ is built from star operations on the components $R_{i}$ 's as in Proposition 3.1, then $\mathfrak{R}$ is a $\star$-Prüfer domain if and only if $R_{i}$ is a $\star_{i}$-Prüfer domain for $\mathcal{U}$-many $i$. We also show that if an ultra star operation $\star_{f}$ on $\mathfrak{R}$ is built from finite type star operations associated to the $\left(\star_{i}\right)_{f}$ 's, then $\mathfrak{R}$ is a $\star_{f}$-Prüfer domain if and only if $R_{i}$ is a Prüfer $\star_{i}$-multiplication domain for $\mathcal{U}$-many $i$.

Recall that given a star operation $\star$ on an integral domain $R$, an ideal $A$ of $R$ is a $\star$-ideal if $A^{\star}=A$. We have the following proposition relating the $\star$-ideals of $\mathfrak{R}$ and the $\star_{i}$-ideals of $R_{i}$ 's.

Proposition 4.1. Let $\star$ be an ultra star operation on $\mathfrak{R}$ built from the star operations $\star_{i}$ 's on $R_{i}$ 's. Then an induced ideal $A=\left(A_{i}\right)_{\mathcal{U}}$ of $\mathfrak{R}$ is $a \star$-ideal if and only if $A_{i}$ is $a \star_{i}$-ideal of $R_{i}$ for $\mathcal{U}$-many $i$. 
Proof. Suppose $A=\left(A_{i}\right)_{\mathcal{U}}$ is a $\star$-ideal of $\mathfrak{R}$. Then $\left(A_{i}\right)_{\mathcal{U}}=\left(\left(A_{i}\right)_{\mathcal{U}}\right)^{\star}=\left(A_{i}^{\star_{i}}\right)_{\mathcal{U}}$. So $A_{i}^{\star_{i}} \subseteq A_{i}$ for $\mathcal{U}$-many $i$, by proposition 2.2 (i). Since $A_{i} \subseteq A_{i}^{\star_{i}}$ for all $i$, it follows that $A_{i}^{\star_{i}}=A_{i}$ for $\mathcal{U}$-many $i$.

Conversely, suppose that $A_{i}^{\star_{i}}=A_{i}$ for $\mathcal{U}$-many $i$. Clearly $A=\left(A_{i}\right)_{\mathcal{U}}=\left(A_{i}^{\star_{i}}\right)_{\mathcal{U}}$ and hence $A$ is a $\star$-ideal.

Given a star operation $\star$ on $R$, one can construct a new star operation $\star_{f}$ as follows: for each $A \in F(R), A^{\star_{f}}=\cup\left\{B^{\star} \mid B \subseteq A\right.$ and $\left.B \in f(R)\right\}$. Note that $d_{f}=d$, where $d$ is the identity star operation and if $\star$ is the $v$-operation we denote $v_{f}:=t$ and call it the $t$-operation. A nonzero ideal $A$ of $R$ is a $\star$-ideal if $A^{\star}=A$. Similarly, we call a $\star$-ideal of $R$ a $\star$-prime ideal of $R$ if it is also a prime ideal. We call a maximal element, if such exists, in the set of all proper $\star$-ideals of $R$ a $\star$-maximal ideal of $R$. We denote $\operatorname{Spec}^{\star}(R)$ the set of all $\star$-prime ideals of $R$ and $\operatorname{Max}^{\star}(R)$ the set of all $\star$-maximal ideals of $R$. An $A \in \mathcal{F}(R)$ is said to be $\star$-invertible if $\left(A A^{-1}\right)^{\star}=R$, whereas a domain $R$ is a Prüfer $\star$-multiplication domain (in short, $\mathrm{P} \star \mathrm{MD})$ if every finitely generated ideal $A$ of $R$ is $\star_{f}$-invertible, i.e., $\left(A A^{-1}\right)^{\star_{f}}=R$ for any $A \in f(R)$. Thus a Prüfer domain is a $\mathrm{P} d \mathrm{MD}$ and $\mathrm{P} v \mathrm{MD}$ is often called a pseudo Prüfer domain.

For lacking a specific reference, we aim to first prove that a domain $R$ is a $\mathrm{P} \star \mathrm{MD}$ if and only if each ideal of $R$ with two generators is $\star_{f}$-invertible. Note that this result is a generalization of the facts that a domain is Prüfer if and only if each ideal with two generators is invertible [12, page 7$]$. The same result has been shown in the $v$-domain ([9]) and in the $\mathrm{P} v \mathrm{MD}([8])$ cases. We start with the recollection of some facts about star operations. Let $\star$ be a star operation on $R$. Recall that $\star$ is stable if $(A \cap B)^{\star}=A^{\star} \cap B^{\star}$. Define $\widetilde{\star}$ by $A^{\widetilde{\star}}:=\cap\left\{A R_{M} \mid M \in \operatorname{Max}^{\star} f(R)\right\}$, for all $A \in F(R)$. Then it is well known that $\widetilde{\star}$ is a stable star operation on $R$ of finite type called the stable star operation of finite type associated to $\star$. It is not hard to see that $\operatorname{Max}^{\widetilde{\star}}(R)=\operatorname{Max}^{\star} f(R)$ [4, Corollary 3.5(2)]. From the latest fact, it then follows that an ideal $A$ is $\widetilde{\star}$-invertible if and only if it is $\star_{f}$-invertible (in fact, if a star operation $\star$ is of finite type, then $\left(A A^{-1}\right)^{\star}=R$ if and only if $A A^{-1} \nsubseteq M$ for all $\left.M \in \operatorname{Max}^{\star}(R)\right)$. From this observation it then follows that $\mathrm{P} \star \mathrm{MD}, \mathrm{P}{ }_{f} \mathrm{MD}$, and $\mathrm{P} \approx \mathrm{MD}$ coincide.

Lemma 4.2. Let $A$ be a finitely generated ideal of an integral domain $R$ and $\star a$ star operation on $R$. If $A$ is $\star_{f}$-invertible, then $A R_{M}$ is principal for every $M \in$ $\operatorname{Max}^{\star}(R)$. 
Proof. Suppose that $A$ is $\star_{f}$-invertible. From the above observation, it follows that $A$ is $\widetilde{\star}$-invertible, i.e., $\left(A A^{-1}\right)^{\widetilde{\star}}=R$. We have, for each maximal $\star_{f}$-ideal $M$, $R_{M}=\left(A A^{-1}\right)^{\widetilde{\star}} R_{M}=\cap\left\{\left(A A^{-1}\right) R_{M} \mid M \in \operatorname{Max}^{\star}(R)\right\} R_{M}=\left(A A^{-1}\right) R_{M}$. Thus $A R_{M}$ is invertible and therefore principal.

Lemma 4.3. Let $R$ be an integral domain and let $\star$ be a star operation on $R$. Then the following statements are equivalent for an integral domain $R$.

(i) $R_{M}$ is a valuation domain for all $M \in \operatorname{Max}^{\star} f(R)$.

(ii) $R$ is a $P \star M D$.

(iii) Every nonzero fractional ideal with two generators is $\star_{f}$-invertible.

Proof. For $(i) \Leftrightarrow(i i)$ [2, Corollary 1.2]. (ii) $\Rightarrow(i i i)$ is clear. So it remains to prove that $(i i i) \Rightarrow(i)$. Let $x, y \in R$, note that if $P$ is a prime ideal of $R$, we have $x R_{P}+y R_{P}=(a, b) R_{P}$ for some $a, b \in R$. But if $P$ is a $\star_{f}$-maximal ideal of $R$ then, by Lemma 4.2 and by hypothesis, $(a, b) R_{P}$ is principal, that is, $R_{P}$ is a valuation domain.

Remark 4.4. Note that the above proof of the preceding lemma is a general version of the proof for the $\mathrm{P} v \mathrm{MD}$ case (see [8, Lemma 1.7]).

Now that we have a bound on finitely generated ideals to characterize $\mathrm{P} \star \mathrm{MD}$, we return to ultraproduct. Recall that an ideal $J$ of a commutative ring is $n$-generated if $J$ can be generated by $n$-elements.

Lemma 4.5. Let $\star$ be the ultra star operation on $\mathfrak{R}$ built from the $\star_{i}$ 's on $R_{i}$ 's. Then an n-generated ideal $A$ is a $a$-invertible ideal of $\mathfrak{R}$ if and only if $A_{i}$ is an $n$-generated $\star_{i}$-invertible ideal of $R_{i}$ for $\mathcal{U}$-many $i$.

Proof. Suppose that $A=\left(A_{i}\right)_{\mathcal{U}}$ is an $n$-generated $\star$-invertible ideal of $\mathfrak{R}$, that is, $\left(A A^{-1}\right)^{\star}=\mathfrak{R}$. Note that since $A$ is $n$-generated, $A_{i}$ is $n$-generated for $\mathcal{U}$-many $i$. So since $A^{-1}=\left(A_{i}^{-1}\right) \mathcal{U}$, we have $\left(A A^{-1}\right)^{\star}=\left(\left(A_{i} A_{i}^{-1}\right)^{\star_{i}}\right) \mathcal{U}=\mathfrak{R}$. Thus $\left(A_{i} A_{i}^{-1}\right)^{\star_{i}}=R_{i}$ for $\mathcal{U}$-many $i$. So $A_{i}$ is an $n$ generated $\star_{i}$-invertible ideal of $R_{i}$ for $\mathcal{U}$-many $i$.

Conversely suppose that $A_{i}$ is $n$-generated and $\left(A_{i} A_{i}^{-1}\right)^{\star_{i}}=R_{i}$ for $\mathcal{U}$-many $i$. Then $\left(\left(A_{i}\right)_{\mathcal{U}}\left(A_{i}^{-1}\right) \mathcal{U}\right)^{\star}=\left(\left(A_{i} A_{i}^{-1}\right)^{\star_{i}}\right) \mathcal{U}=\left(R_{i}\right)_{\mathcal{U}}=\mathfrak{R}$.

Remark 4.6. Let $\star_{i}$ 's be star operations on $R_{i}$ 's. Consider $\left(\star_{i}\right)_{f}$ be the star operation of finite type associated with each $\star_{i}$. If we denote $\star_{f}$ the ultra star operation on $\mathfrak{R}$ built from the $\left(\star_{i}\right)_{f}$ 's (note that $\star_{f}$ is just a notation, so does not mean $\star_{f}$ is the finite type associated to $\star$ ), then using Lemma 4.5, we have that an $n$-generated ideal $A$ is a $\star_{f}$-invertible ideal of $\mathfrak{R}$ if and only if $A_{i}$ is an $n$-generated $\left(\star_{i}\right)_{f}$-invertible ideal of $R_{i}$ for $\mathcal{U}$-many $i$. 
Let us recall that for a general star operation $\star$, a $\star$-Prüfer domain is an integral domain in which every finitely generated nonzero ideal is $\star$-invertible, equivalently every two generated nonzero ideal is $\star$-invertible, see [1, Theorem 2.2]. Note then that a $\mathrm{P} \star \mathrm{MD}$ is a $\star_{f}$-Prüfer domain.

Proposition 4.7. Let $\star$ be the ultra star operation on $\mathfrak{R}$ built from $\star_{i}$ 's on $R_{i}$ 's and $\star_{f}$ be the ultra star operation on $\mathfrak{R}$ built from finite type star operations $\left(\star_{i}\right)_{f}$ associated with each $\star_{i}$. Then

(i) $\mathfrak{R}$ is a $a$-Prüfer domain if and only if $R_{i}$ is a $\star_{i}$-Prüfer domain for $\mathcal{U}$-many $i$.

(ii) $\mathfrak{R}$ is $a \star_{f}$-Prüfer domain if and only if $R_{i}$ is a $P \star_{i} M D$ for $\mathcal{U}$-many $i$.

Proof. Lemma 4.3 states that a domain is a $\mathrm{P} \star \mathrm{MD}$ if and only if every nonzero fractional ideal with two generators is $\star_{f}$-invertible. By Lemma 4.5 (Resp. Remark 4.6), every 2-generated ideal $A$ of $\mathfrak{R}$ is $\star$-invertible (Resp. $\star_{f}$-invertible) if and only if $A_{i}$ is a 2 -generated $\star_{i}$-invertible (Resp. a 2-generated $\left(\star_{i}\right)_{f}$-invertible) ideal of $R_{i}$ for $\mathcal{U}$-many $i$. Hence the proposition follows from [1, Theorem 2.2], Lemma 4.3, and Los' Theorem.

As a consequence of Proposition 4.7, we obtain [10, Proposition 2.2 (iii)] in the case $\star=d$ and more if $\star=v$ and $\star=t$.

Corollary 4.8. Let $\star$ be the ultra star operation on $\mathfrak{R}$ obtained from the $\star_{i}$ 's on $R_{i}$ 's.

(i) Case $\star_{i}=d$. $\mathfrak{R}$ is a Prüfer domain if and only if $R_{i}$ is a Prüfer domain for $\mathcal{U}$-many $i$.

(ii) Case $\star_{i}=v . \Re$ is a $v$-Prüfer domain if and only if $R_{i}$ is a $v$-Prüfer domain for $\mathcal{U}$-many $i$.

(iii) Case $\star_{i}=t$. $\mathfrak{R}$ is a $v_{f}$-Prüfer domain if and only if $R_{i}$ is a PvMD for $\mathcal{U}$-many $i$.

Acknowledgement. The author wishes to express his gratitude to Hans Schoutens for providing some arguments and comments that were helpful in building this paper. The author also thanks the referee for a careful reading of the paper and helpful comments.

\section{References}

[1] D. D. Anderson, D. F. Anderson, M. Fontana and M. Zafrullah, On v-domains and star operations, Comm. Algebra, 37(9) (2009), 3018-3043. 
[2] D. F. Anderson, M. Fontana and M. Zafrullah, Some remarks on Prüfer $\star-$ multiplication domains and class groups, J. Algebra, 319(1) (2008), 272-295.

[3] G. W. Chang, Prüfer *-multiplication domains, Nagata rings, and Kronecker function rings, J. Algebra, 319(1) (2008), 309-319.

[4] M. Fontana and K. A. Loper, Nagata rings, Kronecker function rings, and related semistar operations, Comm. Algebra, 31(10) (2003), 4775-4805.

[5] R. Gilmer, Multiplicative Ideal Theory, Corrected reprint of the 1972 edition, Queen's Papers in Pure and Applied Mathematics, 90, Queen's University, Kingston, ON, 1992.

[6] E. G. Houston, S. B. Malik and J. L. Mott, Characterizations of $\star-$ multiplication domains, Canad. Math. Bull., 27(1) (1984), 48-52.

[7] C. Huneke and I. Swanson, Integral Closure of Ideals, Rings, and Modules, London Mathematical Society Lecture Note Series, 336, Cambridge University Press, Cambridge, 2006.

[8] S. Malik, J. L. Mott and M. Zafrullah, On t-invertibility, Comm. Algebra, 16(1) (1988), 149-170.

[9] J. L. Mott, B. Nashier and M. Zafrullah, Contents of polynomials and invertibility, Comm. Algebra, 18(5) (1990), 1569-1583.

[10] B. Olberding and S. Saydam, Ultraproducts of commutative rings, Commutative Ring Theory and Applications (Fez, 2001), Lecture Notes in Pure and Appl. Math., Dekker, New York, 231 (2003), 369-386.

[11] B. Olberding and J. Shapiro, Prime ideals in ultraproducts of commutative rings, J. Algebra, 285(2) (2005), 768-794.

[12] H. Prüfer, Untersuchen über Teilbarkeitseigenshafen in Körpern, J. Reine Angew. Math., 168 (1932), 1-36.

[13] H. Schoutens, The Use of Ultraproducts in Commutative Algebra, Lecture Notes in Mathematics, 1999, Springer-Verlag, Berlin, 2010.

Olivier A. Heubo-Kwegna

Department of Mathematical Sciences

Saginaw Valley State University

7400 Bay Road

University Center, MI 48710, USA

e-mail: oheubokw@svsu.edu 